SFB

A simple nonparametric test for structural change in joint tail probabilities

Walter Krämer, Maarten van Kampen

Nr. 4/2009

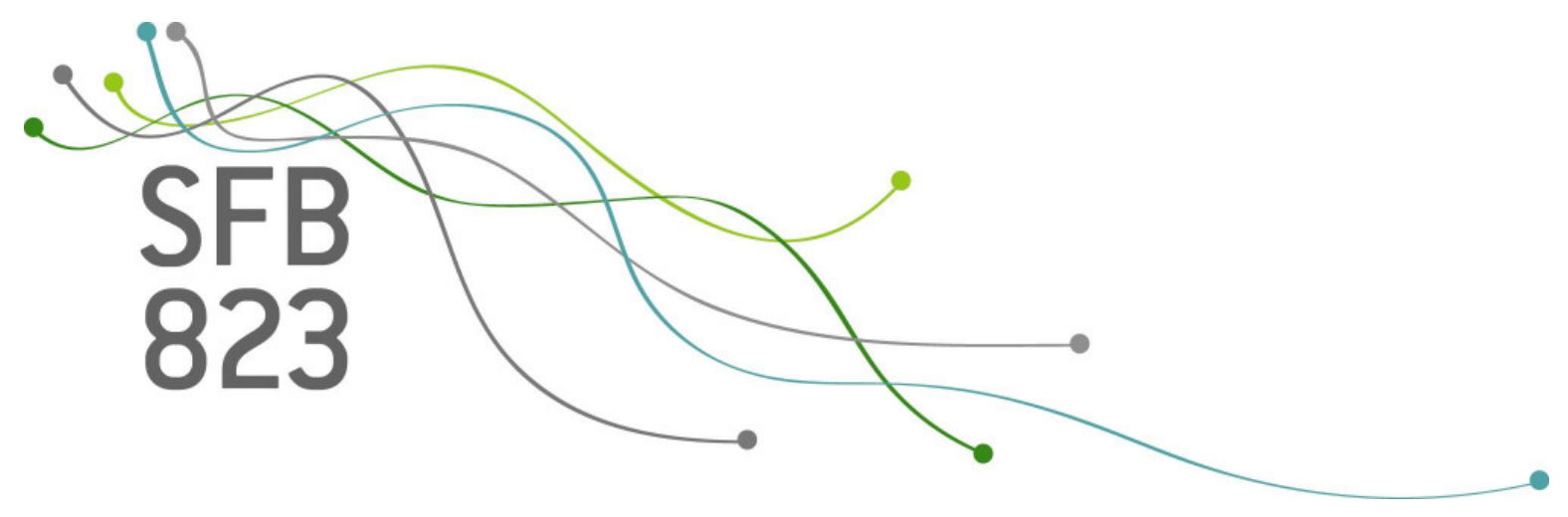





\title{
A simple nonparametric test for structural change in joint tail probabilities ${ }^{1}$
}

\author{
by \\ Walter Krämer \\ Fakultät Statistik, Universität Dortmund \\ D-44221 Dortmund, Germany \\ walterk@statistik.uni-dortmund.de \\ and \\ Maarten van Kampen \\ Ruhr Graduate School in Economics, D-45128 Essen, Germany \\ Maarten.vanKampen@rwi-essen.de
}

Version Juli 2009

\section{Summary}

We propose a new test against a change in the probability of multivariate tail events. The test is based on partial sums of a suitably defined indicator function and detects abrupt changes in joint tail probabilities better than a previously suggested competitor.

Keywords: joint extremes, structural change.

JEL numbers: C12, C14, G15

\footnotetext{
${ }^{1}$ Research supported by Deutsche Forschungsgemeinschaft (DFG). Van Kampen also thanks the Ruhr Graduate School for the financial support.
} 


\section{Introduction and Summary}

In 2008, all major stock markets in the world fell by roughly $40 \%$. The exact figures are: DJIA -33.8\%, S\&P $500-38.1 \%$, CAC40 -42.6\%, FTSE $100-28.7 \%$, DAX $-40.2 \%$ and the MSCI world Index $-40.6 \%$. In fact, the only markets to go up in 2008 were Ghana, Tunisia and Ecuador. The year before, there were likewise some extreme events, with China for instance growing by $96 \%$, but the dependence among markets appeared to be much weaker. Even when focussing on downturns in the market, the joint behavior of stocks in 2008 appears to be unique in recent history. For instance, even though Germany went down even further in 2002 (by -44\%), this downturn was not then shared by others to the same extent. The question therefore arises whether this can be explained by chance or whether there was a structural change in joint tail probabilities sometime in between.

It is important to distinguish two issues here. The first is a possible asymmetry of dependence in the upper and lower tails of a joint distribution. It has by now been firmly established that joint stock returns exhibit larger dependence in the lower than in the upper tail (Ang \& Chen (2002), Fortin \& Kuzmicz (2002), Vaz de Melo Mendez (2005), Sun et al. (2008), among others). This implies that joint downside moves are more probable than joint upside moves even when there is no structural change at all. The second issue is a change in the dependence structure itself, as for instance investigated by Campbell et al. (2002) and Forbes \& Rigobon (2002). And it is this problem which we address in the present paper.

Following Busetti \& Harvey (2008), we base our test on joint exceedences of certain quantiles of the marginal distributions. Instead of using sum of squares of a normalized indicator function, we propose two alternative test statistics. The first is based on the maximum of cumulative sums of the indicator variables (in the spirit of Ploberger \& Krämer (1992)). The second test statistic uses the range of the cumulative sums. We show via Monte Carlo simulation that no test is uniformly superior to the other. While the sum of squares version is more likely to detect gradual or continuous changes in probabilities, the range 
test is more successful with abrupt changes. None of the tests requires prior knowledge as to when a structural change occurs.

\section{The test and its asymptotic null distribution}

Following Busetti \& Harvey (2008), we let $\xi(\tau)$ denote the $\tau$-quantile of some univariate probability distribution. To avoid unnecessary notational complications, we consider continuous distributions only, so $\xi(\tau)$ is uniquely defined. For a bivariate series $y_{1 t}$ and $y_{2 t}, t=1, \ldots, T$, let $\tilde{\xi}\left(\tau_{1}\right)$ and $\tilde{\xi}\left(\tau_{2}\right)$ denote the respective empirical quantiles, and let $C_{T}\left(\tau_{1}, \tau_{2}\right)$ be the proportion of observation where both $y_{1 t}$ and $y_{2 t}$ are less than or equal to $\tilde{\xi}\left(\tau_{1}\right)$ or $\tilde{\xi}\left(\tau_{2}\right)$, respectively. $C_{T}\left(\tau_{1}, \tau_{2}\right)$ is an estimator of $p:=P\left(y_{1 t} \leq \xi\left(\tau_{1}\right), y_{2 t} \leq \xi\left(\tau_{2}\right)\right)$, which is assumed constant under our null hypothesis. For simplicity, we let $\tau_{1}=\tau_{2}=\tau$ from now on.

In applications, one is usually interested mostly in negative tail events, so typical values of $\tau$ or $1 \%$ or $5 \%$. Of course, the whole analysis extends to positive tail events, by reversing the inequality signs, and by taking $\tau=95 \%$ or $\tau=99 \%$, and even more generally, to any changes in the copula of $y_{1}$ and $y_{2}$. In fact, our test may be viewed as a procedure to check the constancy of a copula at a particular point. For concreteness, we stick to the lower tail in the present paper, which is what practitioners are mostly concerned about.

The basic input of our test is what Busetti \& Harvey (2008) call the $\tau$-biquantic, defined as

$$
B I Q_{\tau}(t)=C_{T}(\tau, \tau)-I\left(y_{1 t} \leq \tilde{\xi}_{1}(\tau), y_{2 t} \leq \tilde{\xi}_{2}(\tau)\right), t=1, \ldots, T
$$

where $I($.$) is the indicator function of the event in parentheses. By definition,$ the $B I Q_{\tau}(t)$ add to zero, and partial sums should not deviate too much from zero if $P\left(y_{1 t} \leq \tilde{\xi}(\tau), y_{2 t} \leq \tilde{\xi}(\tau)\right)$ remains constant across the sample. On the other hand, if this probability changes at $t=t^{*}$, say, then the $B I Q_{\tau}(t)$ will tend to be positive up to $t^{*}$ when the probabilities decreases, and the $B I Q_{\tau}(t)$ will tend to be negative when the probability increases. In both cases, the 
cumulated sum of the $B I Q_{\tau}(t)$ will move away from zero farther than can be expected under the null hypothesis. This motivates our choice of test statistic, which is a suitably normalized version of

$$
B_{\tau}(T):=\max _{t=1, \ldots, T}\left|\sum_{i=1}^{t} B I Q_{\tau}(i)\right| .
$$

We show below that, under the null and whenever the events $\left(y_{1 t} \leq \xi(\tau), y_{2 t} \leq\right.$ $\xi(\tau))$ and $\left(y_{1 s} \leq \xi(\tau), y_{2 s} \leq \xi(\tau)\right)$ are independent for all $t \neq s$, the stochastic process

$$
B_{T}(s):=\frac{1}{\sqrt{T C_{T}(\tau, \tau)\left(1-C_{T}(\tau, \tau)\right)}}\left[\sum_{i=1}^{T s} B I Q_{\tau}(i)\right](0 \leq s \leq 1)
$$

tends in distribution to a Brownian Bridge as $T \rightarrow \infty$, so the limiting null distribution of

$$
\frac{1}{\sqrt{T C_{T}(\tau, \tau)\left(1-C_{T}(\tau, \tau)\right)}} B_{\tau}(T)
$$

is identical to that of the Kolmogorov-Smirnov test (see Ploberger \& Krämer $(1992))$. Some useful critical values are 1,22 $(\alpha=10 \%), 1,36(\alpha=5 \%)$ and 1,63 $(\alpha=1 \%)$ where $\alpha$ denotes the significance level. The assumption of independence can be relaxed, as is also shown below.

An alternative test statistic can be derived by examining the range of the $B I Q_{\tau}(t)$, in the spirit of Krämer \& Schotman (1992). In that case, the asymptotic null distribution of the test statistic

$$
\frac{1}{\sqrt{T C_{T}(\tau, \tau)\left(1-C_{T}(\tau, \tau)\right)}}\left[\max _{t=1, \ldots, T} \sum_{i=1}^{t} B I Q_{\tau}(i)-\min _{t=1, \ldots, T} \sum_{i=1}^{t} B I Q_{\tau}(i)\right]
$$

is given by

$$
P(X \leq x)=1+2 \sum_{k=1}^{\infty}\left(1-4 k^{2} x^{2}\right) \exp \left(-2(k x)^{2}\right)
$$

(see e.g. Kennedy (1976)). Some useful critical values are $1.620(\alpha=10 \%)$, $1.747(\alpha=5 \%)$ and $2.001(\alpha=1 \%)$.

Of course, other functionals of the $B I Q_{\tau}(t)$ such as the sum of absolute values might also be used as test statistics, but we focus here on the performance of 
the maximum and the range statistic (compared to the sum of squares statistic proposed by Busetti \& Harvey (2008)).

The convergence in distribution to a Brownian Bridge of $B_{\tau}(s)$ is probably best seen by first considering

$$
\widetilde{B I Q}_{\tau}(t)=p-I\left(y_{1 t}<\xi_{1}(\tau), y_{2 t}<\xi_{2}(\tau)\right)
$$

This is an i.i.d. sequence with zero expectation and finite higher moments of all orders and variance $\sigma^{2}=p(1-p)$, so, by standard results from probability theory (see e.g. Billingsley (1986))

$$
\widetilde{B}_{T}(s):=\frac{1}{\sqrt{T \sigma^{2}}} \sum_{i=1}^{T s} \widetilde{B I Q}(i)
$$

tends in distribution to a standard Wiener Process, and

$$
B_{T}^{*}(s):=\widetilde{B}_{T}(s)-\frac{1}{\sqrt{T \sigma^{2}}} \sum_{i=1}^{T} B \tilde{I} Q(i)
$$

tends in distribution to a Brownian Bridge. The convergence to a Brownian Bridge of $B_{T}(s)$ then follows from the fact that $C_{T}(\tau, \tau)\left(1-C_{T}(\tau, \tau)\right)$ is consistent for $\sigma^{2}=p(1-p)$ and

$$
\max _{t=1, \ldots, T}\left|\sum_{i=1}^{t} B I Q_{\tau}(i)-\sum_{i=1}^{t} B \tilde{I} Q_{\tau}(i)\right| \stackrel{p}{\rightarrow} 0
$$

as $T \rightarrow \infty$.

In empirical applications, when $y_{1 t}$ and $y_{2 t}$ are for instance stock returns, the events $\left(y_{1 t}<\xi(\tau), y_{2 t}<\xi(\tau)\right)$ and $\left(y_{1 s}<\xi(\tau), y_{2 s}<\xi(\tau)\right)$ are in general not independent. In particular, given that $\left(y_{1 t}<\xi(\tau), y_{2 t}<\xi(\tau)\right)$ has occurred, with $\tau$ in the range of $1 \%-5 \%$, the conditional probability of $\left(y_{1, t+1}<\xi(\tau), y_{2, t+1}<\xi(\tau)\right)$ will in general be larger than its unconditional probability due to the well known GARCH-effect.

One way to get around this problem is to replace the variance estimator

$$
\hat{\sigma}^{2}=C_{T}(\tau, \tau)\left(1-C_{T}(\tau, \tau)\right)
$$


by some autocorrelation-consistent version (see e.g. Busetti \& Harvey (2008, p13)) and to invoke the weak dependence of the joint tail events to show that $B_{T}(s)$, properly adjusted, still tends to Brownian Bridge. Another probability is to first fit a GARCH-model to $y_{1 t}$ and $y_{2 t}$ separately and then apply our test to the standardized innovations obtained from these models.

\section{Some finite sample Monte Carlo evidence}

Following Busetti \& Harvey (2008), we examine the performance of the proposed test statistics using simulated values from the Gaussian and the Clayton copula. We explicitly analyze the effect of multiple breaks in the copula parameter. The results in this section and the subsequent section are generated using Ox (see Doornik (2005)) and the G@ARCH package of Laurent \& Peters (2006).

Suppose that there are $m$ breakpoints denoted by $t_{1}, \ldots, t_{m}$. Let $\theta_{j}$ denote copula parameter on segment $j=1, \ldots, m+1$. The bivariate time series $y_{1 t}$ and $y_{2 t}, t=t_{j-1}+1, \ldots, t_{j}$ are drawn from a bivariate Gaussian distribution with correlation $\theta_{j}$ or a Clayton copula $C\left(u, v ; \theta_{j}\right)$ with dependence parameter $\theta_{j}$.

In our base case scenario, we simulate 50000 replications of time series consisting of 3000 observations. In the simulation we restrict the number of copula parameters such that $\theta_{1} \equiv \theta_{2 k+1}$ and $\theta_{2} \equiv \theta_{2 k}, k=0,1, \ldots$ Intuitively, the series consist of periods of low dependence and periods of high dependence. Finally, we apply the test statistics to the $0.05,0.1,0.25$ and 0.50 quantile.

In the Gaussian simulation $\theta_{1}$ equals 0.5 and $\theta_{2}$ takes respectively the values $0.1,0.25,0.5,0.75$ and 0.9 . Table 2 shows that the test proposed by Busetti \& Harvey (2008) slightly outperforms our test statistics if there is a single break in the copula parameter. However, the power of our test statistics is higher in case of two structural breaks. The statistic based on the range outperforms the statistic based on the maximum value. Notice also that the maximum and 
range test are conservative; the power remains in the lower quantiles below 0.05 if the copula is constant.

We also examined the sensitivity of our results by reducing the number of observations and by altering the time point of the structural break. For smaller samples the range statistic retains some power if the break in the correlation parameter is sufficiently high. Reducing the length of the second segment also reduces the power of the test statistics. Finally, the test statistics do not have much power at the $1 \%$ quantile. For sake of brevity we excluded the results of our sensitivity analysis.

The Clayton copula takes the values $\theta_{1}=1$ and $\theta_{2}=1,2.5,7.5$ and 15, respectively. Table 3 shows the simulation results. Again the proposed test statistics outperform the test statistic of Busetti \& Harvey (2008) if there are multiple breaks in the series. The results of the sensitivity analysis are in line with the results of the Gaussian simulation.

\section{An application to stock returns}

We examine daily observations from the Kuala Lumpur stock exchange in Malaysia and the Hang-Seng index in Hong-Kong. The data has been obtained from Econstats and consists of daily observations from December, 7, 1993 trough May, 18, 2009. The corresponding return series is calculated as $y_{t}=$ $\log \left(x_{t} / x_{t-1}\right) \times 100$, where $x_{t}$ denotes the index at time $t=1, \ldots, T$. In the analysis below we only keep the dates at which both return series are observed.

For each series we estimated an $\mathrm{AR}(1)-\mathrm{GARCH}(1,1)$ model where the errors are Student-t distributed. Therefore, the model specification becomes

$$
\begin{aligned}
y_{t} & =\mu+\phi \mu_{t-1}+\varepsilon_{t} \\
\varepsilon_{t} & =z_{t} \sigma_{t} \\
\sigma_{t}^{2} & =\omega+\alpha \varepsilon_{t-1}^{2}+\beta \sigma_{t-1}^{2}
\end{aligned}
$$

where $z_{t}$ is student distributed with $v$ degrees of freedom. 
Subsequently, the different tests are applied to the standardized innovation series. Since the standardized residuals still contain serial correlation, we replaced the variance of the BIC series by a long-run estimator with 36 lags. The number of lags is based on the bandwidth rule $b=4(T / 100)^{1 / 4}$. Table 1 shows that only the range-test is able to reject the null-hypothesis for the 0.1 quantile (using a 5\% significance level). Furthermore, only the square test does not reject $H_{0}$ for the 0.75 quantile. Finally, all tests do reject the null hypothesis for the median.

Table 1: Test statistics based on standardized innovations of the AR(1)GARCH-t $(1,1)$ model.

\begin{tabular}{lllllll}
\hline & \multicolumn{3}{c}{ standard } & \multicolumn{3}{c}{ variance (36 lags) } \\
$\tau$ & Squares & Maximum & Range & Squares & Maximum & Range \\
\hline 0.05 & 0.3360 & 1.1810 & $1.8438^{(b)}$ & 0.2884 & 1.0943 & $1.7084^{(c)}$ \\
0.10 & $0.3672^{(c)}$ & 1.1270 & $1.9766^{(b)}$ & 0.3197 & 1.0516 & $1.8443^{(b)}$ \\
0.25 & 0.2545 & $1.3861^{(b)}$ & $1.8244^{(b)}$ & 0.2047 & $1.2431^{(c)}$ & $1.6361^{(c)}$ \\
0.50 & $0.5670^{(b)}$ & $1.5278^{(b)}$ & $2.0420^{(a)}$ & $0.4906^{(b)}$ & $1.4212^{(b)}$ & $1.8995^{(b)}$ \\
0.75 & $0.7588^{(a)}$ & $2.0686^{(a)}$ & $2.4876^{(a)}$ & $0.4546^{(c)}$ & $1.6011^{(b)}$ & $1.9254^{(b)}$ \\
0.90 & 0.1564 & 1.1789 & $1.7389^{(c)}$ & 0.1158 & 1.0143 & 1.4962 \\
0.95 & 0.1925 & 0.9007 & 1.5315 & 0.1440 & 0.7788 & 1.3243 \\
\hline
\end{tabular}

Table shows test statistics using standard variance estimate of BIC series and a long run variance estimate based on 36 lags. Significance is denoted by the superscripts $1 \%\left({ }^{a}\right), 5 \%$ $\left({ }^{b}\right)$ and $10 \%\left({ }^{c}\right)$.

To examine the stability of our results we also calculated the results using $b=0$ (i.e. standard variance of BIC series). In that case only the range test rejects the null hypothesis at the 0.05 and 0.1 quantile, while only the square test is not able to reject the null hypothesis at the 0.25 quantile. Finally, all tests reject the null hypothesis for the median and the 0.75 quantile. 


\section{References}

Ang, A. \& Chen, J. (2002), 'Asymmetric correlations of equity portfolios', Journal of Financial Economics 63(3), 443-494.

Billingsley, P. (1986), Convergence of Probability Measures, New York, Wiley.

Busetti, F. \& Harvey, A. (2008), When is a copula constant? a test for changing relationships, Cambridge Working Papers in Economics 0841, Faculty of Economics, University of Cambridge.

Cambell, R., Koedijk, C. \& Kofman, K. (2002), 'Increased correlation in bear markets', Financial Analysts Journal .

Cambell, R., Koedijk, C. \& Kofman, K. (2008), 'Increasing correlations or just fat tails?', Journal of Empirical Finance 58, 87-94.

Doornik, J. A. (2005), Object-Oriented Matrix Programming Using Ox, 4 edn, London: Timberlake Consultants Press.

Forbes, K. J. \& Rigobon, R. (2002), 'No contagion, only interdependence: Measuring stock market comovements', Journal of Finance 57(5), 22232261.

Fortin, I. \& Kuzmicz, C. (2002), 'Tail dependence in stock returns', Journal of Intelligent Systems in Accounting, Finance and Management 11, 89-107.

Jondeau, E. \& Rockinger, M. (2006), 'The copula-garch model of conditional dependencies: An international stock market application', Journal of International Money and Finance 25(5), 827-853.

Kennedy, D. (1976), 'The distribution of the maximum Brownian excursion', Journal of Applied Probability 13, 371-376.

Krämer, W. \& Schotman, P. (1992), 'Range vs. maximum in the ols-based version of the cusum test', Economics Letters 40(4), 379-381.

Laurent, S. \& Peters, J. P. (2006), G@RCH 4.2, Estimating and Forecasting ARCH Models, London: Timberlake Consultants Press. 
Ploberger, W. \& Krämer, W. (1992), 'The cusum test with ols residuals', Econometrica 60(2), 271-85.

Rodriguez, J. C. (2007), 'Measuring financial contagion: A copula approach', Journal of Empirical Finance 14(3), 401-423.

Sun, W., Rachev, S., Stoyanov, S. \& Fabozzi, F. (2008), 'Multivariate skewed student's t copula in the analysis of nonlinear and asymmetric dependence in the german equity market', Studies in Nonlinear Dynamics $\&$ Econometrics $12(2), 1572-1572$.

Vaz de Melo Mendez, B. (2005), 'Asymmetric extreme interdependence in emerging equity markets', Applied Stochastic Models in Business and Industry 21, 89-107. 
Table 2: Gaussian Copula with structural breaks in correlation: simulated rejection frequencies $\left(\mathrm{T}=3000, \operatorname{Rep}=50000, \theta_{1}=0.5\right)$

\begin{tabular}{|c|c|c|c|c|c|c|c|c|}
\hline$m$ & test & $\tau$ & $\theta_{2}$ & 0.10 & 0.25 & 0.50 & 0.75 & 0.90 \\
\hline \multirow{4}{*}{1} & \multirow{4}{*}{ Squares } & 0.05 & & 0.68 & 0.35 & 0.05 & 0.46 & 0.93 \\
\hline & & 0.10 & & 0.90 & 0.53 & 0.05 & 0.65 & 0.99 \\
\hline & & 0.25 & & 0.98 & 0.71 & 0.05 & 0.80 & 1.00 \\
\hline & & 0.50 & & 0.95 & 0.63 & 0.05 & 0.76 & 1.00 \\
\hline \multirow{4}{*}{1} & \multirow{4}{*}{ Maximum } & 0.05 & & 0.65 & 0.31 & 0.04 & 0.44 & 0.93 \\
\hline & & 0.10 & & 0.90 & 0.51 & 0.04 & 0.64 & 0.99 \\
\hline & & 0.25 & & 0.98 & 0.71 & 0.05 & 0.80 & 1.00 \\
\hline & & 0.50 & & 0.96 & 0.63 & 0.05 & 0.76 & 1.00 \\
\hline \multirow{4}{*}{1} & \multirow{4}{*}{ Range } & 0.05 & & 0.48 & 0.20 & 0.03 & 0.31 & 0.84 \\
\hline & & 0.10 & & 0.80 & 0.37 & 0.04 & 0.50 & 0.97 \\
\hline & & 0.25 & & 0.95 & 0.57 & 0.04 & 0.68 & 1.00 \\
\hline & & 0.50 & & 0.91 & 0.49 & 0.04 & 0.63 & 1.00 \\
\hline \multirow{4}{*}{2} & \multirow{4}{*}{ Squares } & 0.05 & & 0.14 & 0.09 & 0.05 & 0.07 & 0.32 \\
\hline & & 0.10 & & 0.23 & 0.11 & 0.05 & 0.13 & 0.57 \\
\hline & & 0.25 & & 0.44 & 0.15 & 0.05 & 0.20 & 0.79 \\
\hline & & 0.50 & & 0.37 & 0.13 & 0.05 & 0.18 & 0.74 \\
\hline \multirow{4}{*}{2} & \multirow{4}{*}{ Maximum } & 0.05 & & 0.19 & 0.11 & 0.04 & 0.11 & 0.39 \\
\hline & & 0.10 & & 0.34 & 0.16 & 0.05 & 0.19 & 0.64 \\
\hline & & 0.25 & & 0.56 & 0.23 & 0.05 & 0.28 & 0.84 \\
\hline & & 0.50 & & 0.49 & 0.20 & 0.05 & 0.26 & 0.81 \\
\hline \multirow{4}{*}{2} & \multirow{4}{*}{ Range } & 0.05 & & 0.31 & 0.14 & 0.04 & 0.27 & 0.78 \\
\hline & & 0.10 & & 0.64 & 0.27 & 0.04 & 0.42 & 0.93 \\
\hline & & 0.25 & & 0.89 & 0.45 & 0.04 & 0.59 & 0.99 \\
\hline & & 0.50 & & 0.82 & 0.40 & 0.04 & 0.53 & 0.98 \\
\hline \multirow{4}{*}{3} & \multirow{4}{*}{ Squares } & 0.05 & & 0.19 & 0.10 & 0.05 & 0.13 & 0.36 \\
\hline & & 0.10 & & 0.33 & 0.15 & 0.05 & 0.19 & 0.55 \\
\hline & & 0.25 & & 0.52 & 0.22 & 0.05 & 0.26 & 0.75 \\
\hline & & 0.50 & & 0.43 & 0.19 & 0.05 & 0.24 & 0.72 \\
\hline \multirow{4}{*}{3} & \multirow{4}{*}{ Maximum } & 0.05 & & 0.16 & 0.09 & 0.04 & 0.12 & 0.38 \\
\hline & & 0.10 & & 0.35 & 0.15 & 0.05 & 0.19 & 0.61 \\
\hline & & 0.25 & & 0.58 & 0.22 & 0.05 & 0.28 & 0.81 \\
\hline & & 0.50 & & 0.49 & 0.20 & 0.05 & 0.26 & 0.78 \\
\hline \multirow{4}{*}{3} & \multirow{4}{*}{ Range } & 0.05 & & 0.12 & 0.06 & 0.03 & 0.09 & 0.29 \\
\hline & & 0.10 & & 0.26 & 0.10 & 0.04 & 0.14 & 0.53 \\
\hline & & 0.25 & & 0.50 & 0.17 & 0.04 & 0.22 & 0.78 \\
\hline & & 0.50 & & 0.41 & 0.15 & 0.04 & 0.20 & 0.75 \\
\hline
\end{tabular}


Table 3: Clayton Copula with structural breaks in dependence: simulated rejection frequencies $\left(\mathrm{T}=3000, \operatorname{Rep}=50000, \theta_{1}=1\right)$

\begin{tabular}{|c|c|c|c|c|c|c|c|}
\hline$m$ & test & $\tau$ & $\theta_{2}$ & 1 & 2.5 & 7.5 & 15 \\
\hline & \multirow{4}{*}{ Squares } & 0.05 & & 0.05 & 0.41 & 0.76 & 0.83 \\
\hline \multirow[t]{3}{*}{1} & & 0.10 & & 0.05 & 0.65 & 0.96 & 0.98 \\
\hline & & 0.25 & & 0.05 & 0.89 & 1.00 & 1.00 \\
\hline & & 0.50 & & 0.05 & 0.87 & 1.00 & 1.00 \\
\hline \multirow{4}{*}{1} & \multirow{4}{*}{ Maximum } & 0.05 & & 0.04 & 0.39 & 0.75 & 0.83 \\
\hline & & 0.10 & & 0.04 & 0.64 & 0.96 & 0.99 \\
\hline & & 0.25 & & 0.05 & 0.89 & 1.00 & 1.00 \\
\hline & & 0.50 & & 0.05 & 0.87 & 1.00 & 1.00 \\
\hline \multirow{4}{*}{1} & \multirow{4}{*}{ Range } & 0.05 & & 0.04 & 0.27 & 0.62 & 0.71 \\
\hline & & 0.10 & & 0.04 & 0.50 & 0.91 & 0.96 \\
\hline & & 0.25 & & 0.05 & 0.80 & 1.00 & 1.00 \\
\hline & & 0.50 & & 0.05 & 0.78 & 1.00 & 1.00 \\
\hline \multirow{4}{*}{2} & \multirow{4}{*}{ Squares } & 0.05 & & 0.05 & 0.07 & 0.17 & 0.20 \\
\hline & & 0.10 & & 0.05 & 0.13 & 0.42 & 0.53 \\
\hline & & 0.25 & & 0.05 & 0.27 & 0.89 & 0.96 \\
\hline & & 0.50 & & 0.05 & 0.25 & 0.97 & 1.00 \\
\hline \multirow{4}{*}{2} & \multirow{4}{*}{ Maximum } & 0.05 & & 0.04 & 0.11 & 0.24 & 0.28 \\
\hline & & 0.10 & & 0.04 & 0.19 & 0.51 & 0.61 \\
\hline & & 0.25 & & 0.05 & 0.37 & 0.92 & 0.97 \\
\hline & & 0.50 & & 0.05 & 0.35 & 0.98 & 1.00 \\
\hline \multirow{4}{*}{2} & \multirow{4}{*}{ Range } & 0.05 & & 0.04 & 0.22 & 0.54 & 0.62 \\
\hline & & 0.10 & & 0.04 & 0.42 & 0.86 & 0.92 \\
\hline & & 0.25 & & 0.04 & 0.71 & 1.00 & 1.00 \\
\hline & & 0.50 & & 0.05 & 0.68 & 1.00 & 1.00 \\
\hline \multirow{4}{*}{3} & \multirow{4}{*}{ Squares } & 0.05 & & 0.05 & 0.12 & 0.24 & 0.27 \\
\hline & & 0.10 & & 0.05 & 0.19 & 0.45 & 0.53 \\
\hline & & 0.25 & & 0.05 & 0.34 & 0.84 & 0.92 \\
\hline & & 0.50 & & 0.05 & 0.32 & 0.94 & 0.99 \\
\hline \multirow{4}{*}{3} & \multirow{4}{*}{ Maximum } & 0.05 & & 0.04 & 0.11 & 0.24 & 0.29 \\
\hline & & 0.10 & & 0.04 & 0.19 & 0.49 & 0.59 \\
\hline & & 0.25 & & 0.05 & 0.37 & 0.89 & 0.95 \\
\hline & & 0.50 & & 0.05 & 0.35 & 0.96 & 1.00 \\
\hline \multirow{4}{*}{3} & \multirow{4}{*}{ Range } & 0.05 & & 0.04 & 0.08 & 0.18 & 0.22 \\
\hline & & 0.10 & & 0.04 & 0.15 & 0.41 & 0.51 \\
\hline & & 0.25 & & 0.04 & 0.30 & 0.87 & 0.95 \\
\hline & & 0.50 & & 0.05 & 0.28 & 0.97 & 1.00 \\
\hline
\end{tabular}



